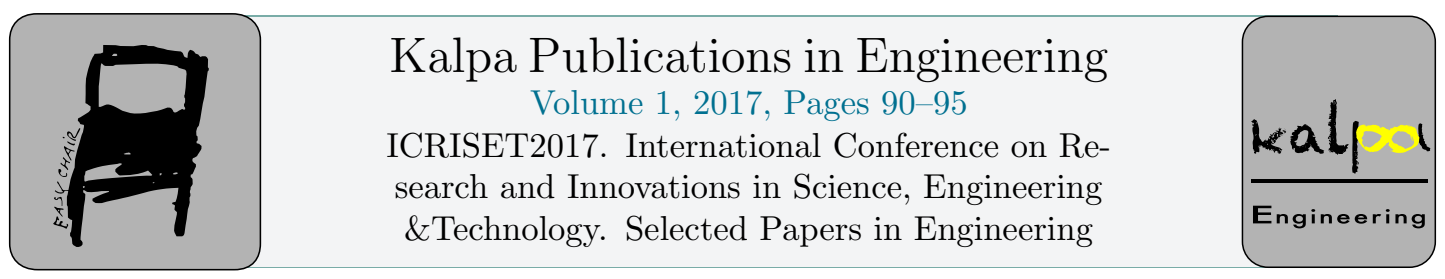

\title{
RPCA, MRA and ICA Methods for Motion Artifact Identification in AECG Signals
}

\author{
${ }^{1}$ Deepak Vala, ${ }^{2}$ Tanmay Pawar \\ ${ }^{1}$ EasyChair \\ ${ }^{1,2}$ Electronics Engineering Department, BVM, V. V. Nagar \\ ${ }^{1}$ dlvala@bvmengineering.ac. in, ${ }^{2}$ tanmay.pawar@gmail.com
}

\begin{abstract}
In this paper, an analysis of RPCA, MRA and ICA methods for motion artifact identification in AECG signals is preformed. First we applied a RPCA to ECG signal with synthesis motion artifact by low-pass filtering random noise signal. In the process, we have verified that the RPCA error magnitude is significantly greater for the noisy episodes as compared to the clean ECG signal portions. We used 25 data-sets from Physionet website and also used recorded AECG of five person of different physical activity for AECG analysis. We used wavelet for AECG signal denoising. and then ICA, technique used for removal of motion artifacts of synthesized ECG data of MIT$\mathrm{BIH}$ and of AECG signals.
\end{abstract}

\section{Introduction}

In this paper, an attempt is made to study the Ambulatory ECG and identification of motion artifacts using different technique. A method based on RPCA (Recursive Principal Component Analysis) is thoroughly applied for identification of artificially induced motion artifacts in various sub-bands on the ECG signals from the MIT-BIH arrhythmia database of physionet [7]. Similarly, the RPCA algorithm is also tested for actually induced motion artifact due to performing various usual physical. A method based on MRA (Multi-resolution Analysis) framework using wavelets is proposed in this paper for identification of motion artifacts in ambulatory ECG signals. We also used ICA to separate motion artifacts from ECG signal [1, 2, 3, and 6]. In this work, digital AECG signals are recorded using a wearable ECG recorder and a bench-top Biopac MP 36 data-acquisition system. Usual common body movement activities like of the healthy persons Hand, Waist, Walking 
movement and Sitting up and down is studied in this work. The effect of the pace of activities is also analyzed by allowing the different pace levels: slow and fast, for four particular healthy persons.

\section{RPCA method}

There are several issues associated while analyzing such an ECG signal contaminated with motion artifacts, e.g. detection, classification and quantification of the motion artifacts (noise). The motion artifacts frequency overlapped with ECG signal frequency, so it is difficult to filter out both components [8]. Pawar et al. [6] proposed an RPCA method to identify motion artifacts due to physical activities.

In this work, as we have to explore the entirely possible bandwidth for motion artifact, $0-10 \mathrm{~Hz}$ using the RPCA-based method, we have considered the low-pass filtered white noise acting as the motion artifact in an ECG signal. We have used the ECG signals available from Physionet websites [7]. The additive motion artifacts are synthesized using low-pass filtered noise with varying signal to noise ratio(SNR) and spectral ranges. The motion artifacts artificially generated by low-pass filtering the random noise signal. A random noise signal filtered with fourth-order Butterworth low-pass filter with different cutoff frequencies, i.e. $5 \mathrm{~Hz}, 10 \mathrm{~Hz}$ has been used as the synthesis motion artifact. This synthesized motion artifact signal, $\mathrm{N}(\mathrm{k})$, is then added to the ECG signal, $\mathrm{S}^{\prime}(\mathrm{k})$, with 10 s of noisy duration and $50 \mathrm{~s}$ of clean duration. [9]. Similar way we applied RPCA to a recorded AECG signal of all physical activities. We also calculated RPCA error mean and variance of physical activities and find that slower activities have a lower average mean and variance compared to faster physical activities, according to table 1 [3].
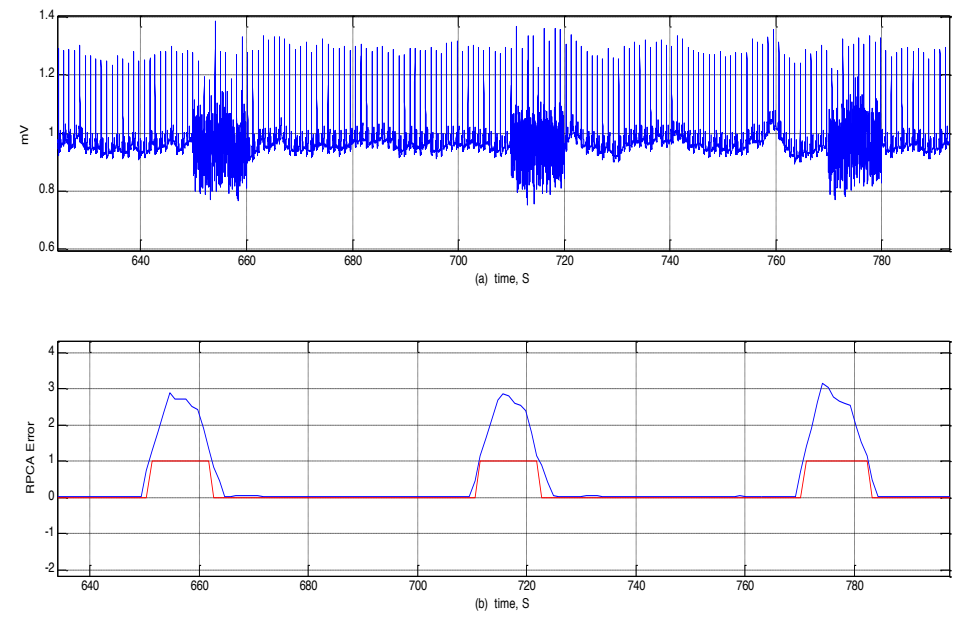

Figure 1: (a) ECG signal and (b) The corresponding RPCA Error for detection of motion artifact episodes for tap \#101 of MIT-BIH database. $\mathrm{B}=0-5 \mathrm{~Hz}, \alpha=0.99$ 
Table 1 RPCA error and variance mean of AECG signal for different movements and different subjects

\begin{tabular}{|r|c|c|c|c|c|}
\hline \multirow{2}{*}{$\begin{array}{c}\text { S } \\
\text { r. } \\
\text { No }\end{array}$} & $\begin{array}{c}\text { Physical } \\
\text { activity }\end{array}$ & \multicolumn{2}{|c|}{ Slow activity } & \multicolumn{2}{|c|}{ Fast activity } \\
\cline { 3 - 6 } & $\begin{array}{c}\text { Hand } \\
\text { Movement }\end{array}$ & 9.15998 & 66.7702 & 14.0313 & 115.918 \\
\hline 1 & $\begin{array}{c}\text { Sitting to } \\
\text { Standing } \\
\text { Movement }\end{array}$ & 18.5363 & 83.0713 & 49.4022 & 137.189 \\
\hline 3 & $\begin{array}{c}\text { Waist } \\
\text { Movement }\end{array}$ & 12.0345 & 166.305 & 23.6193 & 224.921 \\
\hline 4 & $\begin{array}{c}\text { Walking } \\
\text { Movement }\end{array}$ & 16.637 & 129.553 & 32.2462 & 186.677 \\
\hline
\end{tabular}

\section{MRA}

Then we used multi-resolution analysis for AECG analysis. There is basic, two wavelet processes, decomposition and reconstruction. Decomposition process gives us low-frequency components. Then we performed reconstruction, or synthesis to get original signal. We use both graphical way and matlab coding to find a motion artifact signal from level eight using bior6. 8 wavelet to reconstruct the coefficients of a one-dimensional signal. Then wavelet function "wrcoef" used, to compute the vector of reconstructed coefficients. We use suitable component to reconstruct motion artifact and AECG signal.

$\mathrm{S}=\mathrm{A} 8+\mathrm{D} 8+\mathrm{D} 7+$

Here $\mathrm{S}$ is a reconstructed signal. Here in equation (1) it is a combination of approximate component A8 reconstructed at level eight and D1 to D8 are components from level one to eight. Here first few components represent the ECG signal. We experimentally found that the detail eighth component is identified motion artifact [4].
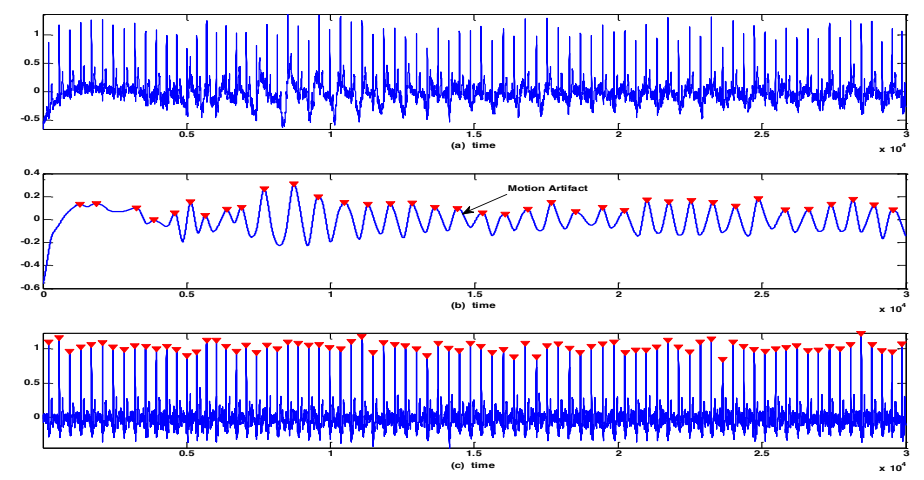

Figure 2: (a) AECG signal with motion artifacts (b) Identified motion artifacts (c) AECG signal with the separated motion artifact as well as its QRS peak detection for right hand up down slow movement 


\section{ICA}

We also used ICA technique [11] for removal of motion artifacts in AECG signal. We have used simulated ECG signal as well as real ECG signal and successfully separated motion artifact and clean ECG signal using ICA. First we have used the ECG signals available from Physionet site. We used synthesis motion artifacts for ICA analysis on 10 ECG data sets. We successfully separated synthesized motion artifact signal using the FastICA algorithm as shown in fig. 3.

We also calculated an ECG-beat count, synthesis motion artifact interval and its total number of peak count as per in table 2. Also we used recorded AECG signal of different healthy persons. That signal is low-pass filtered and smoothen using a moving average filter. We used derived filter signal as a second source signal shown in fig. 4. The mixtures are separated using the FastICA algorithm to obtain motion artifact and ECG signal as shown in fig.4(e) . Similar way, we identified and removed motion artifact of for all types of physical activities [5].
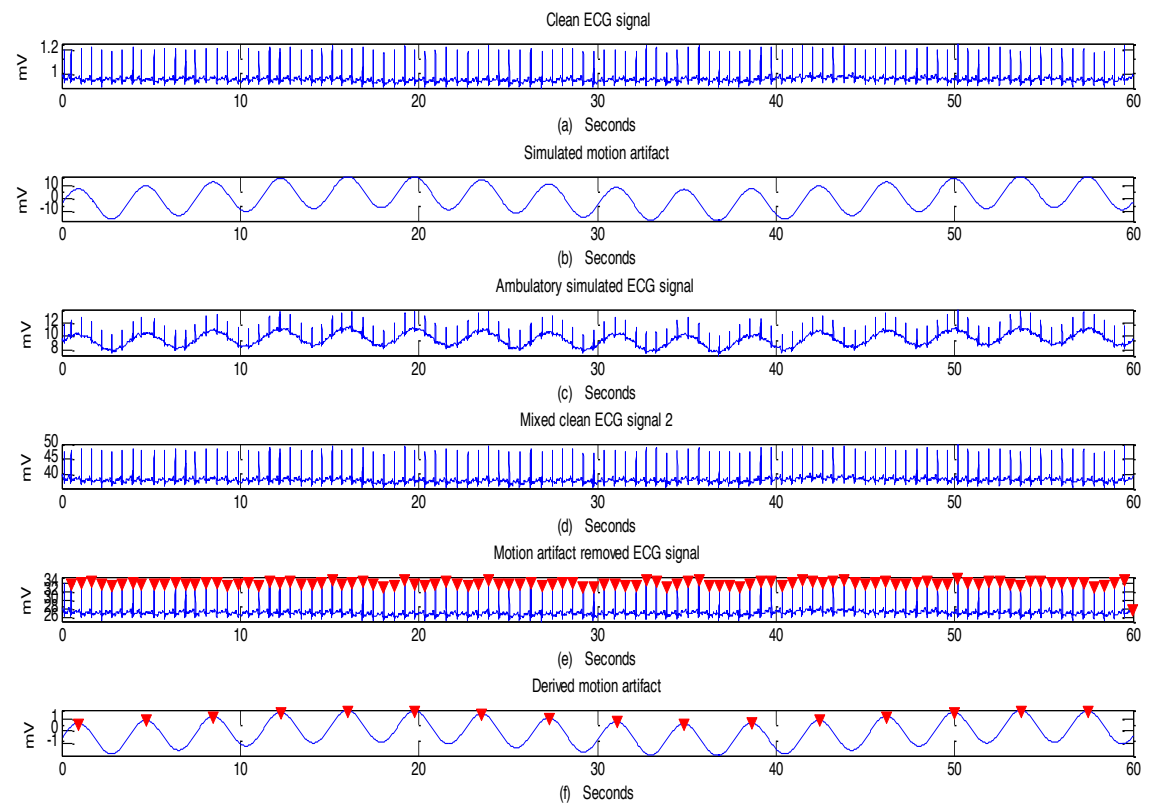

Figure. 3: ICA analysis of slow synthesis signal (a) clean ECG signal (b) Simulated motion artifact (c) Ambulatory simulated ECG signal (d) Corrupted ECG signal 2(e) Motion artifact removed signal (f) motion artifact signal 

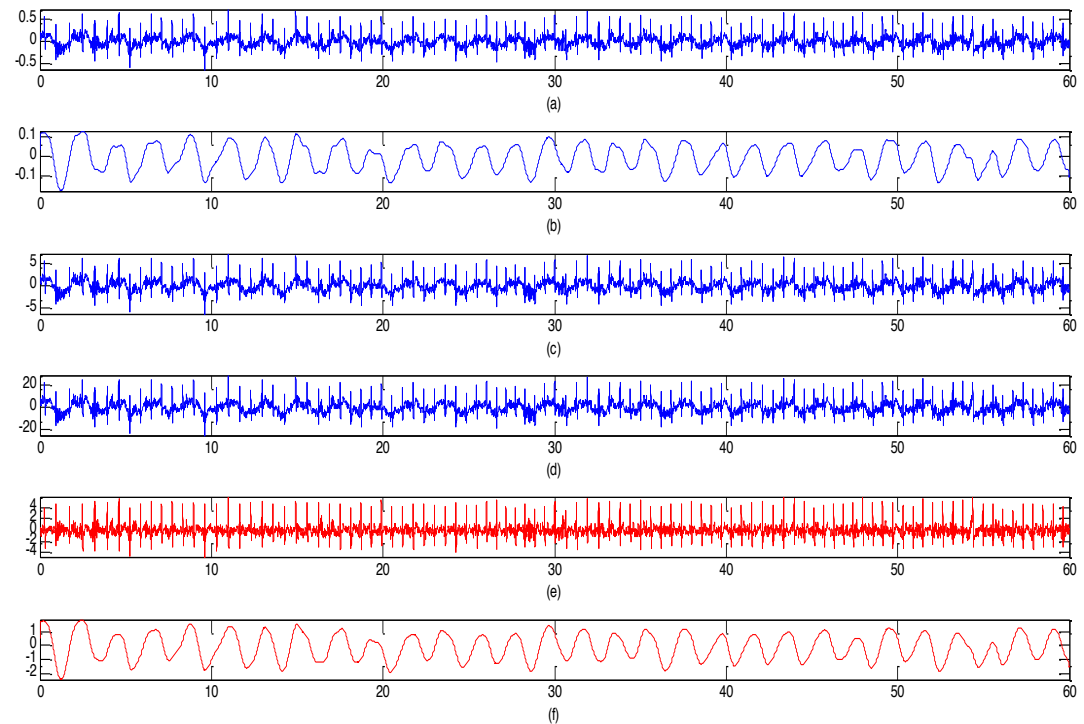

Figure 4: Identification of motion artifact in slow hand movement (a) Ambulatory ECG signal (b) filtered ECG signal (c) Corrupted ECG signal 1 (d) Corrupted ECG signal 2 (e) Motion artifact removed signal (f) motion artifact signal

Table 2 Peak intervals, No. of Peak, hearty beat results for slow and fast movement activity [10, 4]

\begin{tabular}{|c|c|c|c|c|c|c|}
\hline Person \# & $\begin{array}{r}\text { Peak } \\
\text { Interval }\end{array}$ & $\begin{array}{r}\text { No. } \\
\text { of peak }\end{array}$ & $\begin{array}{l}\text { Heart } \\
\text { beat }\end{array}$ & $\begin{array}{r}\text { Peak } \\
\text { Interval }\end{array}$ & $\begin{array}{r}\text { No. } \\
\text { of peak }\end{array}$ & $\begin{array}{l}\text { Heart } \\
\text { beat }\end{array}$ \\
\hline \multirow{7}{*}{$\begin{array}{l}\text { Mean of } \\
\text { five } \\
\text { persons }\end{array}$} & \multicolumn{3}{|c|}{ Slow walking movement } & \multicolumn{3}{|c|}{ Fast walking movement } \\
\hline & 903 & 33 & 100 & 609 & 50 & 105 \\
\hline & \multicolumn{3}{|c|}{ Slow waist movement } & \multicolumn{3}{|c|}{ Fast waist movement } \\
\hline & 1132 & 26 & 94 & 680 & 41 & 107 \\
\hline & \multicolumn{3}{|c|}{ Slow sitting to standing movement } & \multicolumn{3}{|c|}{$\begin{array}{c}\text { Fast sitting to standing } \\
\text { movement }\end{array}$} \\
\hline & 1039 & 29 & 99 & 782 & 38 & 104 \\
\hline & \multicolumn{3}{|c|}{ Slow hand movement } & \multicolumn{3}{|c|}{ Fast hand movement } \\
\hline Mean & 836 & 37 & 76 & 650 & 45 & 78 \\
\hline $\begin{array}{l}\text { Total } \\
\text { mean }\end{array}$ & 977 & 31 & 92.25 & 680 & 43 & 98.50 \\
\hline
\end{tabular}




\section{Conclusion:}

We have presented an offline analysis of the RPCA method to identifying a motion artifact in an ECG signal. We have taken the offline 25 ECG signal from of physionet website. We have verified that the RPCA error magnitude is significantly greater for the noisy episodes as compared to the clean ECG signal portions. We have used the wavelet function to separate out motion artifacts from AECG signal of four types of physical body movements of common daily routine activities of five persons. We also used independent component analysis to separate motion artifacts from ECG signal.

\section{References:}

Vala D. and T Pawar. "A Survey on ambulatory ECG and identification of motion artifact." International Journal of Engineering Research and Development 1.7 (2012): 3841.

Kher, R., Vala, D., Pawar, T., \& Thakar, V. K. (2010, April). Ambulation study of ECG in wearable devices. In Proceedings of the 2009 International Conference on Signals, Systems and Automation (ICSSA 2009). Universal-Publishers.

3 Kher, Rahul, Dipak Vala, Tanmay Pawar, and Vishvjit Thakar. "RPCA-based detection and quantification of motion artifacts in ECG signals." Journal of medical engineering \& technology 37, no. 1 (2013): 56-60.

$4 \quad$ Vala, Deepak, Tanmay Pawar, and V. K. Thakar. "Identification of Motion Artifact in Ambulatory ECG Signal Using Wavelet Techniques." American Journal of Biomedical Engineering 3.6 (2013): 194-198.

5 Deepak Vala, Tanmay Pawar, V. K. Thakar, "Motion Artifact removal in Ambulatory ECG Signal using ICA", June 14 Issue6, International Journal on Recent and Innovation Trends in Computing and Communication (IJRITCC), ISSN: 2321-8169, PP: 1457 - 1461

Chaudhuri, Subhasis, Tanmay D. Pawar, and Siddhartha Duttagupta. Ambulation analysis in wearable ECG. Springer Publishing Company, Incorporated, 2009.

MIT-BIH Arrhythmia database (mitdb) [cited 2012 May 24]. Available at: www.physionet.org

T. Pawar, S. Chaudhuri and S. Duttagupta. "Analysis of Ambulatory ECG Signal”, In 28th international conference of the IEEE on engineering in medicine, New York, NY pp. $3094-3097$, Aug. 2006.

9 Kher, Rahul, Deepak Vala, and Tanmay Pawar. "Detection of Low-pass Noise in ECG Signals." In National Conference on Recent Trends in Engineering \& Technology, V V Nagar, 13-14 May 2011.

10 Deepak Vala, Tanmay Pawar, V. K. Thakar "Identification and Analysis of Motion Artifact in Ambulatory ECG Signal in 1-D and 2-D" International Journal of Emerging Technology and Advanced Engineering, Volume 3, Issue 9, ISSN 2250-2459, September 2013

11 Naik, Ganesh R., and Dinesh K. Kumar. "An overview of independent component analysis and its applications." Informatica: An International Journal of Computing and Informatics 35.1 (2011): 63-81. 